\title{
Determining Effective Parameters on CO Concentration in Tehran Air by Sensitivity Analysis based on Neural Network Prediction
}

\author{
F. Qaderi ${ }^{1}$, E. Babanezhad ${ }^{1}$, M. Ebrahimi Ghadi ${ }^{2}$ \\ 1. Faculty of Civil Engineering, Babol Noshirvani University of Technology, Babol, Iran \\ 2. Sirjan University of Technology, Sirjan, Iran
}

Received: 22 September 2019; Accepted: 22 October 2019; Available online: 30 November 2019

\begin{abstract}
One of the most toxic pollutant gases produced by fossil fuels is carbon monoxide. Hence, the accurate and regular estimation and control of $\mathrm{CO}$ in the cities such as Tehran is inevitable. In this research, for the first time, CO concentration in ambient air was predicted based on 12 important urban and meteorological parameters by neural network. Also, the sensitivity analysis of the factors that effect on the concentration of carbon monoxide in Tehran was investigated based on the pollutant concentration predictive model. In this research, the daily statistical data of Tehran metropolis over the course of five consecutive years from 12 factors affecting the amount of carbon monoxide in Tehran, such as population, density, precipitation, temperature, urban traffic, wind speed, gasoil consumption, moisture, air flow, effective vision and air pressure was used. Based on this database, the artificial neural network with the best possible algorithm had been trained to predict this contaminant and root mean square error of model was equal to 2.54. Then, sensitivity analysis was done to find the most effective factor on the concentration of carbon monoxide, urban density and air pressure. In order to control this hazardous contaminant in urban management, these parameters should be taken into account. Based on the result, by preventing the construction of high towers in Tehran, wind speed average will increase and increasing in wind speed (25\%) caused to reducing in carbon monoxide concentration (about 12\%). Also, prevention of urban density (25\%) will cause to prevention of increasing CO concentration (about 10\%).
\end{abstract}

Keywords: Carbon monoxide; Sensitivity analysis; Artificial neural network.

\section{Introduction}

Experiments and prediction in environmental issues are important. Water, wastewater and air pollutions are the main subjects of environmental engineering. Many researchers study this subject in recent years. Air pollution has become an undeniable problem in many countries. The world's policy makers, especially in the United States and Europe, have drafted international laws and treaties to reduce air pollution [1-3]. The rapid growth of countries' economies through industry and non-compliance with the international laws have exposed these countries into air pollution as a global environmental challenge [4]. One of the toxic and dangerous air pollutants is carbon monoxide, produced by incomplete combustion of hydrocarbons [5]. The sources of entry of this greenhouse gas can be different sources like as the car exhausts, steam boilers, smoke and tobacco and incorrect use of generators and charcoal furnaces [6, 7].

This highly dangerous gas can have a great destructive impact on human health, for example, its hazardous effects on respiration can be noted [8-10]. Tehran is the largest city in Iran. It also is polluted due to the expansion of residential areas caused by urbanization and rapid population growth [11, 12].

In recent years, artificial neural network was used in environmental phenomena prediction [13]; especially in air quality prediction. Boznar et al. first predict $\mathrm{SO}_{2}$ concentration by this method in Slovenia industrial are as in the early 1990s [14]. Then, after studying the relationship between carbon monoxide dispersion and transport parameters using artificial neural network, Dorzdowicz in 1997 presented a model for predicting the CO concentration using an artificial neural network [5]. In addition, Gardner and Dorling, in 1997, used a multi-layered perceptron neural network in the central part of London to predict nitrogen oxide and nitrogen dioxide concentrations [15].

In the past researches, the effect of different parameters on the dependent environmental variables have been investigated [16-21]. A lot of studies were conducted on sensitivity analysis to determine the effect of each parameter. For instance, a study by Banimahd et al (2005) used the sensitivity analysis for the soil behavior and obtained acceptable results [22]. Moreover, Yeh (2007) conducted research on the modeling of slump flow in concrete using second-order regressions and artificial neural networks, this research showed that artificial neural 
network models can be used for numerical experiments and sensitivity analysis can be done on the parameters by this methods [23].

According to previous research, the simultaneous effect of urban parameters and meteorological parameters on the air pollution has not been studied so far. Previous research only provided an air pollution prediction model with limited factors. Also, they did not determine the important factors affecting carbon monoxide concentration.

In this paper, for the first time, 12 urban and meteorological parameters affecting the CO concentration in Tehran metropolis are investigated. The 12 important parameters affecting the air pollution studied in this paper are urban traffic, rainfall, population, air humidity, wind speed, gasoline and gasoil consumption, air pressure, temperature, urban density, air traffic, and urban green space. In this study, using data collected on a daily basis for 5 years by stations located in the city predicts the carbon monoxide concentration through artificial neural network. In this study, carbon monoxide concentration modeling was designed with the least error, and the closest model was presented to the reality. For the first time, the factors influencing the increase of CO concentration have been determined by sensitivity analysis to reduce the amount of this toxic and dangerous pollutant.

\section{Materials and methods}

\subsection{Location}

Some previous environmental papers worked on case studies [24,25]. In this research Tehran was selected as a case study. Tehran is located at 35 degrees 35 minutes to 35 degrees and 48 minutes of North latitude and 51 degrees and 17 minutes to 51 degrees 33 minutes of East longitude with an area of about 800 square kilometers and with 22 municipalities. The population of Tehran is over 8 million. This city is bounded from the North to the central Alborz Mountains and from the South to the Northwest margins of the central desert of Iran, and is bounded from the East to Jajrood Valleys and from the West to Karaj Valleys.

\subsection{Data collection}

One of the most important issues in the artificial neural network is the provision of the initial data for network training, and the ability of the neural network to respond to the new issues largely depends on the initial data. Therefore, it is necessary to collect data that is relevant in terms of the mechanism to the desired output and also to the extent that the network is well trained so that it can have sufficient generalization power. In research papers, the data can be prepared from the experiments in laboratory or the site [18, 26-28]. As a result, the data have been collected daily for five consecutive years. In this study, 12 parameters affecting the increase of carbon monoxide concentration in Tehran have been investigated. These include urban traffic, rainfall, population, moisture, wind speed, gasoline and gasoil consumption, air pressure, temperature, urban density, air traffic, and urban green space. The statistical data has been collected from 2012 to 2016. These 12 parameters are statistically categorized into two categories of urban parameters like as population, density, green space, etc., and meteorological parameters like as temperature, air pressure, wind speed, etc. Urban parameters have been collected daily from Tehran's Central Statistical Center and meteorological parameters were collected by Tehran weather stations. Furthermore, the CO concentration monitored by Tehran Air Quality Control Company has been monitored and collected daily.

\subsection{Data normalization}

In some previous researches, the data have been investigated or normalized before modeling in order to reaching to the best optimization or prediction [29-31]. Some parameters affecting the CO concentration change in different seasons, such as rainfall, temperature and moisture, and since data is recorded on a daily basis, data input is normalized to help training of artificial neural network. Data normalization is a process to standardize it. In this way, the data will be matched to each other. This method is only suitable for the use in and modeling smart methods, since during the training of the smart grids, the numerical learning rate is diminished, and if output of the model changes are not significant in a few rounds of training, the learning process stops. Many of the techniques used to test the statistical assumptions, such as regression and parametric tests, have several initial assumptions. If these assumptions are not met, the results cannot be guaranteed with the accuracy desired. One of the most important assumptions is to have a normal distribution of an independent or dependent variable in the absence of which, the use of statistical techniques is endangered. Assume that by using graphing methods and good fit tests, this assumption was checked and the normalization of some variables was rejected. However, we have a variable that doesn't have a normal distribution and we want to convert it with the appropriate method to transform the observations of the variable normally distributed. If the assumption of normalization is rejected, at first, the pertinent observations or errors in the data should be identified. To detect the pertinent observations, Chebyshev Boxed and Inequal Diagrams or Tests of Distribution Free can be used. After the removal of the pertinent observations, data distribution may be normalized immediately. Therefore, data normalization was done by BoxCox transformation. 


\subsection{Artificial neural network}

An artificial neural network is built on the basis of a computational unit called perceptron. Scientists have introduced artificial neuron by natural neuron Inspiring. Three basic components of weight (w), bias (b) and the stimulus (f) function are in neural network. These three components are unique to each neuron, using this artificial neuron to respond to its inputs.

The algorithm used in this research is to model the feed-forward back propagation problem which is shown in Figure 1 as a schematic image of this algorithm. In an artificial neuron, depending on the problem, we can use various propulsion functions that can be linear or nonlinear. The function used in this research is LOGSIG. This function is expressed by the general equation 1 .

$$
a=f(n)=\frac{1}{1+e^{-c n}}, \quad c>0
$$

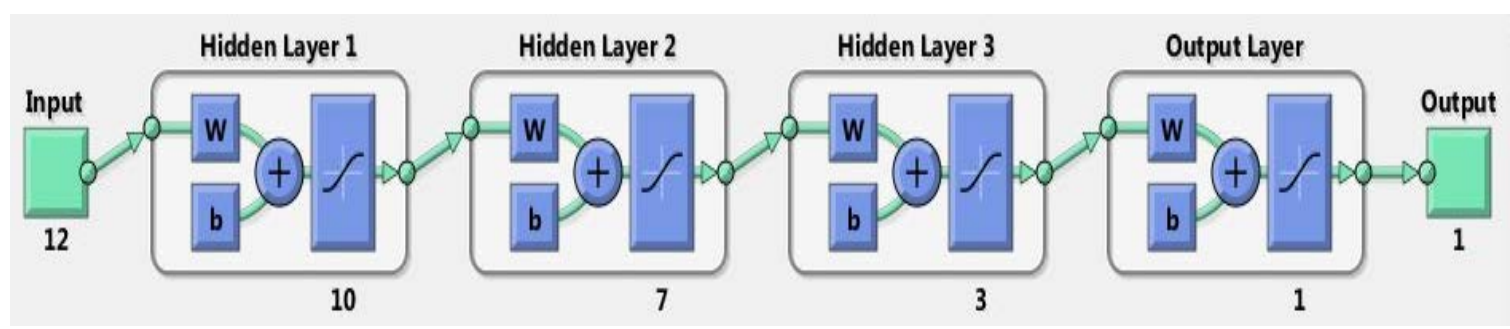

Figure 1. Feed-forward back propagation Algorithm of ANN

In this research, the data are divided into two dependent and independent categories. Air pollution data introduced as dependent data and 12 parameters affecting carbon monoxide concentration introduced as independent data were reported daily to the network. In order to train the neural network, $10 \%$ of the data was separated. The rest was used as neural network input, which was trained to training, validation, and TEST categories with ratios of 75, 15 and 15 percent. There are also statistical indicators like Mean Absolute Error (MAE), Random Error $\left(\mathrm{R}^{2}\right)$, Root Mean Square Error (RMSE) and Mean Squared Error (MSE) used for evaluating performance of network, in learning process [2] .

\subsection{Sensitivity analysis}

The purpose of the sensitivity analysis is to examine the rate of output variation for minor variations in the input data. As presented in the proposed algorithm, the search has been done to select an optimal neural network with an appropriate response from the perspective of the estimation error. In order to analyze the sensitivity of the network, the following process has established the results of which are presented. At first, the network selection was performed with a number of specific layers, then some data was selected as random samples of the test. In the next step, for the simulation, performance and output tests, an estimation was done for each item of the test data. Then, each of the characteristics of the test data with the normal distribution function was audible. There were 40 test data in this study, and each data element had 20 parameters that affect air pollution. Then, in order to verify the output sensitivity to curve 1 in the table of the values of the 40 items, $40 \times 100$ new values were generated. These values are with average initial values of $10 \%$. A neural network was then evaluated for every 4,000 new samples, and the deviation of new responses or initial network responses was obtained for 40 test data. This amount of distortion will be plotted in terms of the percentage of noise entered into the input. This process is repeated for the 20 transition parameters affecting the Tehran's air pollution and the sensitivity of the network is plotted for changes in each characteristic. Equation 2 represents the function used in sensitivity analysis.

$$
\begin{aligned}
& y^{n}=f\left(x_{1}+x_{2}+x_{3}+\ldots \ldots+x_{12}\right) \\
& y^{n}+\Delta y_{j}^{n}=f\left(X_{1}^{n}+\Delta X_{1 j}^{n}+X_{2}^{n}+\ldots \ldots+X_{12}^{n}\right)
\end{aligned}
$$

where y represents the amount of contamination, $\Delta X= \pm 10 \% X_{n}, j=1-100$ Represents the number of random reproduced. $x_{1}$ is traffic, $x_{2}$ is temper, $x_{3}$ is precipitation, $x_{4}$ is humidity, $x_{5}$ is wind speed, $x_{6}$ is visibility, $x_{7}$ is petrol, $\mathrm{x}_{8}$ is gasoline, $\mathrm{x}_{9}$ is airport, $\mathrm{x}_{10}$ is population, $\mathrm{x}_{11}$ is dense and $\mathrm{x}_{12}$ is pressure.

\section{Discussion and results}

Normally, the logarithmic transformation makes the normal data distribution. This is clearly apparent from the four examples of histogram data in Figure 2. The volume of data in this study was very high, it made it possible to checking histograms of data and normalized graphs or QQ Plot for normalization. Diagram A represents the 
wind speed, which indicates that the maximum wind speed is between 0.8 and 1.3 meters per second, Diagram B represents the air pressure in Tehran, with the highest statistical pressure of 6.76 and Diagram $C$ represents the moisture of the city of Tehran, monitored daily by observation stations over the course of five consecutive days. As can be seen, the highest moisture density is between 3.2 and 3.8.
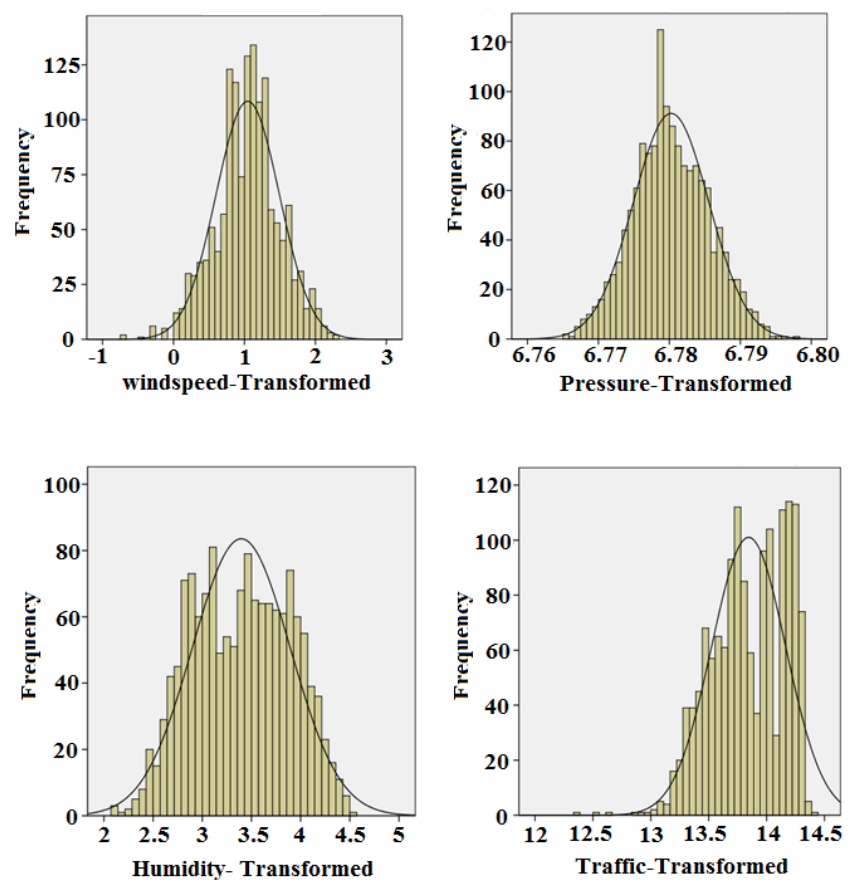

Figure 2. Illustration of Histogram diagram, traffic, humidity, wind speed and fuel

Figure 3 shows the amount of carbon monoxide concentration changes for 5 consecutive years. These changes were collected by 45 air pollution monitoring stations. The closer stations to the city center, the higher the concentration is as a result of crowded traffic and traffic jams. As it can be see, in October, CO concentration increases considerably, due to the sudden drop in temperature in Tehran and the arrival of winter. And the lowest carbon monoxide concentrations are in May each year. The average carbon monoxide concentration is $36.61 \mathrm{ppm}$ in 2012, 38.52 ppm in 2013, 40.22 ppm in 2014, 38.73 ppm in 2015, and 40.21 ppm in 2016. Thus, this diagram represents the fact that in the cold seasons, the CO concentration increases, which actually increases with the decrease of the temperature and decreases with the increase of the temperature.

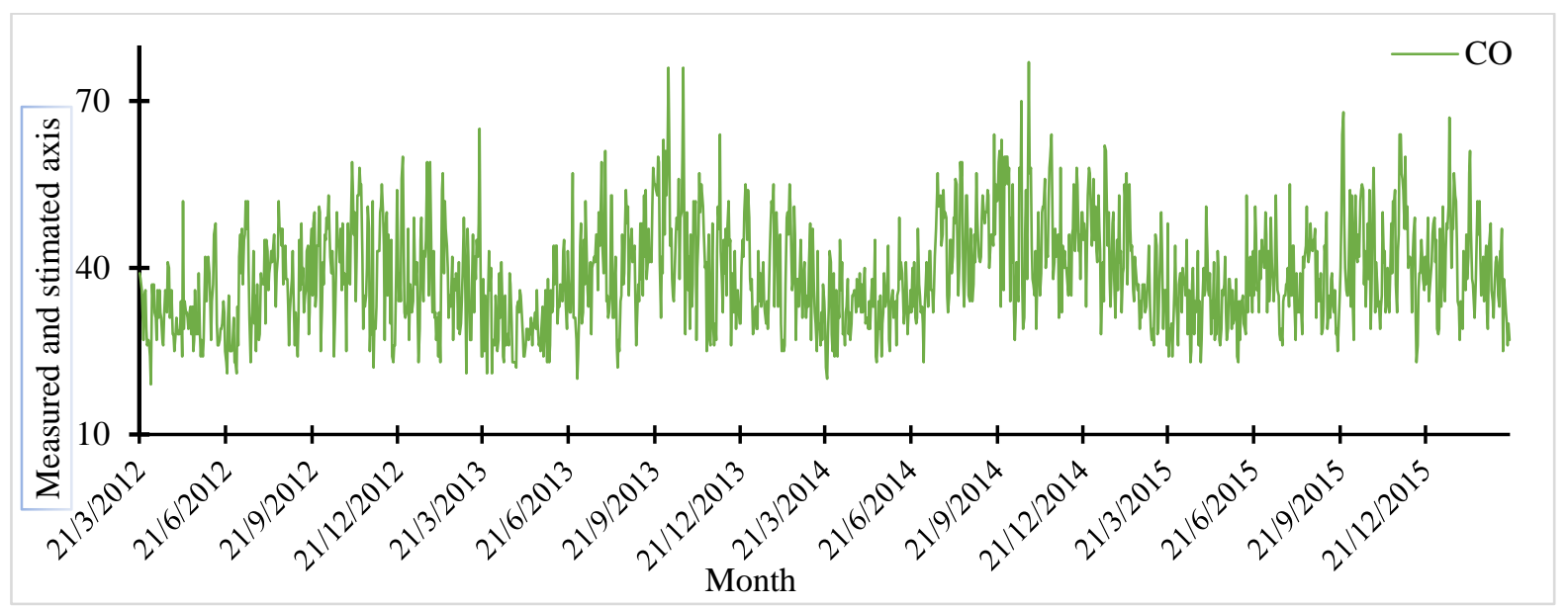

Figure 3. CO concentration changes for 5 years period

Modeling of carbon monoxide concentration was done using artificial neural network. It this research, the feedforward back propagation was used. Then, repeated attempts were done and errors were made in selecting the number of intermediate layers and the number of neurons to select the optimum neural network for CO concentration prediction, and in each layer, different functions such as TanSig, LogSig and Pureline were 
performed. The MAE, $\mathrm{R}^{2}$, RMSE and MSE errors were extracted for each test performed in the function and number of different neurons. Among the 415 tests, the lowest error was found to be 2.54 . Among the 415 tests, the feed-forward back propagation algorithm with TanSig function and 3 intermediate layers was selected and 10 neurons were in the first hidden layer, seven neurons were in the second hidden layer and the third hidden layer had three neurons. This mode has lowest error value. As a result, this network is the best way for prediction of CO concentration in the Tehran air.

As shown in Figure 4, in the process of training, the network first has been trained with regard to input parameters, then, the average square error of the error has decreased and the generalization power has also increased. When the network begins to fix an error to a constant value, this progress goes downward because the goal is to increase the generalization power. Where the network begins to fix the errors to a constant value, the training should be disconnected, and is the method of crossover weighting. Epoch's number is specified in horizontal axis. In each epoch, the inputs are initially applied and the result is created, and then error values are calculated and the correction in network parameters were done based on error rate.

In Figure 5, the time series of carbon monoxide concentration is observed and time series caused by model is shown in the time interval of test data and network accuracy is seen, obviously. Error rate of best training of network, were respectively $0.17,0.03,0.13,0.99$ for $\mathrm{R}^{2}$, MAE, RMSE and MSE. This model is predicted CO concentrations in Tehran, carefully.

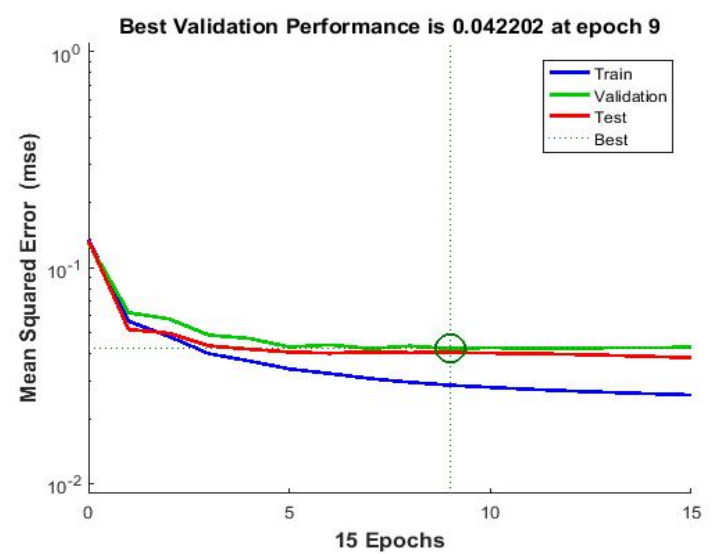

Figure 4. Result of training of neural network in Train, Validation and Test

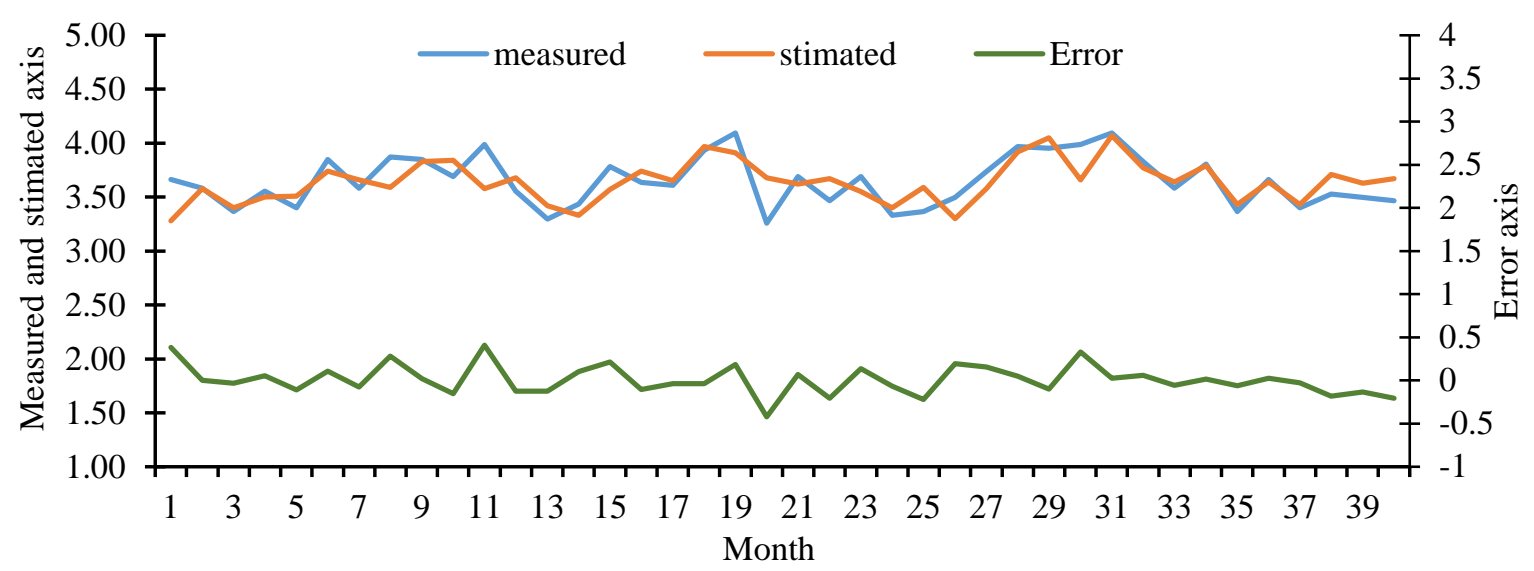

Figure 5. Comparison between CO concentration in model and real values

According to Figure 6, the most suitable network was obtained with three hidden layers and with a correlation coefficient of 0.663 . There were 10 neurons in the first layer, seven neurons in the second layer and three neurons in the third layer, respectively. To obtain this result, coding in MATLAB environment was used. In Figure 5, the horizontal axis of the measured data is observed on the vertical axis of the predicted data by the neural network based on the inputs.

Figure 7 consists of 12 graphs, and each of which shows the sensitivity of various parameters affecting the air pollution of Tehran. The horizontal axis of the graphs represents the magnitude of the percentage of variations in each of parameters. All parameters were reconstructed randomly and with a normal distribution with a mean of 0 and a standard deviation of $10 \%$. Then, in each parameter, the output value is obtained for the reconstructed data 
and compared with the initial output, and the percentage of the error estimate is shown in the vertical axis. As it can be seen, the amount of rainfall, effective vision and temperature are low due to the fact that the rate of change between the initial data and the reconstructed data is very low and the graph has changed linearly, which has the least effect on the Tehran's pollution. In Graph 3, the parameters of wind speed, air pressure and density parameters have not only changed nonlinearly, but also the degree of variation in the vertical axis, which indicates the error rate between the initial data and the reconstructed data, is very high, resulting in the highest sensitivity. They have the highest effect on Tehran's air pollution.

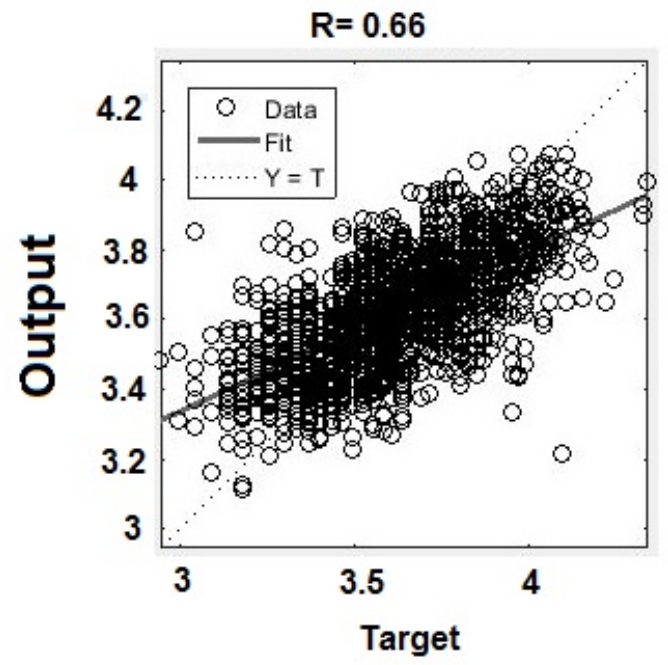

Figure 6. Distribution of predicted CO concentration through the model
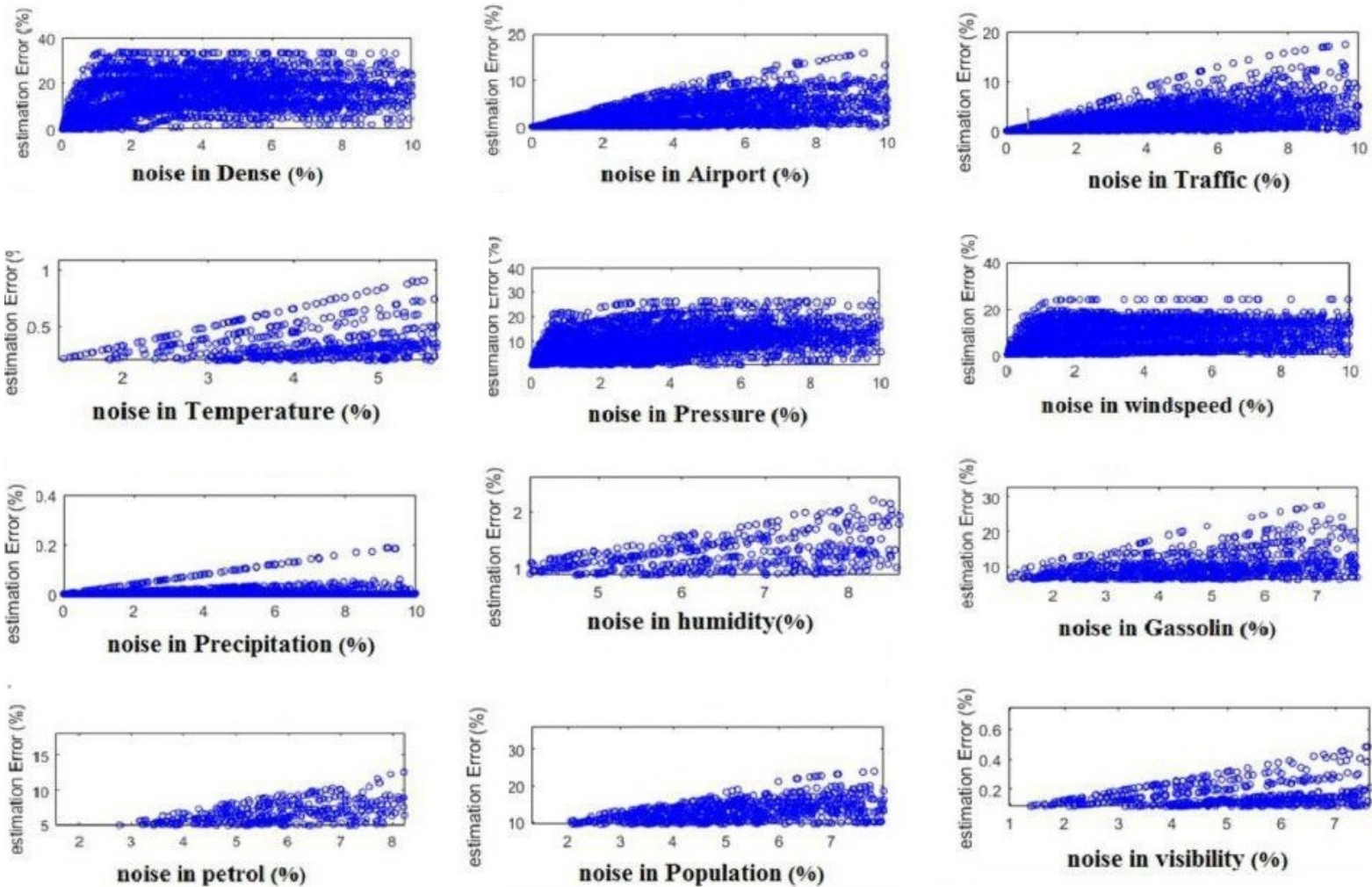

Figure 7. Sensitivity analysis of parameters affecting Tehran’s air pollution

As stated earlier, wind speed, air pressure, and density have the most effect on the pollution of Tehran. Figure 8 and Table 1 show the increase of the wind speed, air pressure, and urban density in Tehran. According to the observations, as the wind speed increases, the $\mathrm{CO}$ concentration decreases so that by increasing the wind speed by $10 \%$, the CO concentration decreases by $9.96 \%$. According to Tehran's meteorological data, the average wind 
speed monitored before the last 10 years has been 2.38 and over the past 10 years, it has been 1.92 meters per second and over the past five years, it has reached to 1.62 meters per second. One of the major causes of wind speed is the construction of uncontested high-rise buildings in Tehran, which is like a barrier to the flow of winds and prevents the increase of wind speed entering the city. Air pressure is one of the most influential parameters of Tehran's air pollution. Considering that the temperature of pollutants is higher than the air temperature on the ground, the pollutant moves upward and to the higher altitudes and this upward movement continues till the air temperature gets higher than the pollutants temperature and at that point, there will be an accumulation of different pollutants. Therefore, with the increase of the height, the air pressure decreases and the concentration level of pollutants gets higher. This graph proves this issue in Tehran. The amount of urban density at hectare level in Tehran is one of the most effective parameters of pollution concentration in Tehran. As it is shown in this graph, with increasing the urban density, the concentration of pollutants also increases, so that by increasing the urban density to ten, twenty-five and fifty percent, the concentration of the pollutants increases by $1.96 \%, 10.81 \%$ and $13.08 \%$, respectively.

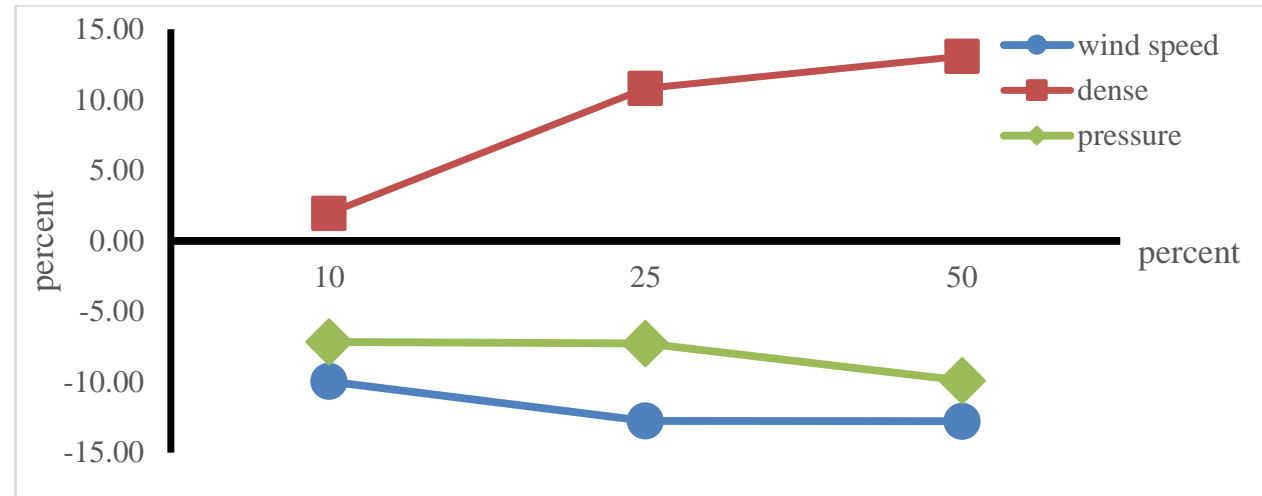

Figure 8. Validation of the most significant parameters of Tehran's air pollution

Table 1. Analysis of the most influential factor in Tehran's air pollution

\begin{tabular}{cccc}
\hline & $\mathbf{\% 1 0}$ & $\mathbf{\% 2 5}$ & $\mathbf{\% 5 0}$ \\
\hline Wind speed & -9.96 & -12.74 & $\mathbf{- 1 2 . 7 7}$ \\
dense & 1.96 & 10.81 & $\mathbf{1 3 . 0 8}$ \\
pressure & -7.16 & -7.25 & $\mathbf{- 9 . 8 9}$ \\
\hline
\end{tabular}

So far, the conducted studies to reduce the air pollution have had several shortcomings so that without following the urban management policies, and preventing urban pollution through risk management and air pollution control, only provided passive and cross-sectional responses to the increase of air pollution. The results of such studies have not been used by the scientific support system in the management decisions to reduce the air pollution. Therefore, due to the importance of reducing the air pollution, the applied analysis of the findings of the artificial modeling and sensitivity analysis of the factors influencing the air pollution increase in Tehran city are presented in Table 2, using daily data obtained from monitoring the CO concentration in 45 air pollution measurement stations in Tehran. Among parameters affecting the concentration of carbon monoxide, 6 parameters are directly controlled and managed by humans. These six parameters include urban density, air traffic, population, gasoline and gasoil consumption and urban density of Tehran. If any of these parameters are properly managed and controlled, the concentration of toxic and hazardous carbon monoxide contaminants in the city will be reduced. Each of these six parameters, respectively, urban density, gasoline consumption, population, gasoil consumption and traffic volume and air traffic have greater sensitivity and effect on increasing the CO concentration. This table increased each of these parameters using the model that was trained in the artificial neural network by $10 \%, 25 \%$ and $50 \%$, and presented the rate of change in carbon monoxide concentration for each parameters, so that if the gasoline consumption in this city increases by $25 \%$, the CO concentration will increase by $7.25 \%$ or if the population of Tehran increases by $10 \%$ in the coming years, the CO concentration will increase by $0.8 \%$. In another case, if the mass production of urban traffic increases by $50 \%$, the CO concentration in the city will increase by $3.45 \%$. Therefore, as a result of the correct and practical method for controlling the CO concentration in Tehran, the parameters should be controlled according to their priority to reduce the concentration of carbon monoxide, to develop the urban management and human social interactions, and make sustainable development in the air pollution of Tehran. The appropriate results of this study are so that this template can be used in the air quality management support system to achieve the ultimate goal of optimal urban transport management in Tehran metropolis. 
Table 2. Analysis of human factors effect on Tehran's air pollution

\begin{tabular}{cccc}
\hline & $\mathbf{\% 1 0}$ & $\mathbf{\% 2 5}$ & $\mathbf{\% 5 0}$ \\
\hline dense & 1.96 & 10.81 & $\mathbf{1 3 . 0 8}$ \\
Gasoline & 0.9 & 7.25 & $\mathbf{8 . 5 4}$ \\
Population & 0.8 & 3.07 & $\mathbf{8 . 0 8}$ \\
Petrol & 0.7 & 1.78 & $\mathbf{3 . 6 0}$ \\
Traffic & 0.5 & 1.6 & $\mathbf{3 . 4 5}$ \\
Airport & 0.66 & 1.54 & $\mathbf{2 . 7 1}$ \\
\hline
\end{tabular}

\section{Conclusion}

Tehran's Air pollution is one of the serious problems in this metropolis. Management issues in metropolises is always based on this phenomenon to prevent its dangerous effects by providing executive and economic solutions. In the present study, to control the CO concentration in Tehran, 12 factors were studied. The data obtained was used as the input data for prediction. A neural network was then used to predict the carbon monoxide concentration and sensitivity analysis was performed on these 12 parameters. The most effective parameters were determined to be wind speed, urban density and air pressure. The study showed that with an increase of only $10 \%$ of the wind speed in the city, 9.96\% of the carbon monoxide concentration reduced, while the skyscrapers and towers reduced about $15.62 \%$ of the wind speed over the past 10 years. As a result, with a municipal administration, it is possible to reduce the $\mathrm{CO}$ concentration by reducing the height of some towers in areas where the inflow of wind dominates the city. Also, the amount of urban density greatly affects the CO concentration. Thus, in the densely populated areas of Tehran, the CO concentration is very high and the research has shown that if the urban density is increased by only 25\%, the CO concentration increases by $10.81 \%$. It causes irreparable damage in the long run. Consequently, in order to control CO concentration in Tehran, it is necessary to pay attention to three factors of urban density and wind speed in the city.

\section{Acknowledgment}

The financial support of this research prepared by the Babol Noshirvani University of Technology (grant number: BNUT/393016/97). The authors thank the Babol Noshirvani University of Technology.

\section{References}

[1] Beckx C, Panis LI, Arentze T, Janssens D, Torfs R, Broekx S, Wets G. A dynamic activity-based population modelling approach to evaluate exposure to air pollution: methods and application to a Dutch urban area. Environmental Impact Assessment Review. 2009;29(3):179-185.

[2] Ghadi ME, Qaderi F, Babanezhad E. Prediction of mortality resulted from $\mathrm{NO}_{2}$ concentration in Tehran by Air Q+ software and artificial neural network. International Journal of Environmental Science and Technology. 2019;16(3):1351-1368.

[3] Qaderi F, Asadi P, Tamadoni A, Azizi M. Evaluation of sustainability of development in zone 22 of Tehran by ecological footprint method. Geography and Development Iranian Journal. 2018;16(50):231-245.

[4] Evans J, van Donkelaar A, Martin RV, Burnett R, Rainham DG, Birkett NJ, Krewski D. Estimates of global mortality attributable to particulate air pollution using satellite imagery. Environmental Research. 2013;120:33-42.

[5] Drozdowicz B, Benz SJ, Santa Cruz AS, Scenna NJ. A neural network based model for the analysis of carbon monoxide concentration in the urban area of Rosario. Air Pollution Modelling, Monitoring and Management. 1997; 15:677-684.

[6] Zhou S, Weitzman M, Vilcassim R, Wilson J, Legrand N, Saunders E, Travers M, Chen LC, Peltier R, Gordon T. Air quality in New York City hookah bars. Tobacco Control. 2015;24(e3):e193-e198.

[7] Iqbal S, Clower JH, Hernandez SA, Damon SA, Yip FY. A review of disaster-related carbon monoxide poisoning: surveillance, epidemiology, and opportunities for prevention. American Journal of Public Health. 2012;102(10):1957-1963.

[8] Samoli E, Touloumi G, Schwartz J, Anderson HR, Schindler C, Forsberg B, Vigotti MA, Vonk J, Košnik M, Skorkovsky J, Katsouyanni K. Short-term effects of carbon monoxide on mortality: an analysis within the APHEA project. Environmental Health Perspectives. 2007;115(11):1578-1583.

[9] Bell ML, Peng RD, Dominici F, Samet JM. Emergency hospital admissions for cardiovascular diseases and ambient levels of carbon monoxide results for 126 united states urban counties, 1999-2005. Circulation. 2009;120(11): 949-955. 
[10] Chen R, Pan G, Zhang Y, Xu Q, Zeng G, Xu X, Chen B, Kan H. Ambient carbon monoxide and daily mortality in three Chinese cities: the China Air Pollution and Health Effects Study (CAPES). Science of the Total Environment. 2011;409(23):4923-4928.

[11] Saadatabadi AR, Bidokhti AA. Urbanization effects on local climate in Tehran Megapolis. Research Journal of Environmental Sciences. 2011;5(1):1-21.

[12] Alizadeh-Choobari O, Ghafarian P, Adibi P. Inter-annual variations and trends of the urban warming in Tehran. Atmospheric Research. 2016;170:176-185.

[13] Qaderi F, Babanezhad E. Prediction of the groundwater remediation costs for drinking use based on quality of water resource, using artificial neural network. Journal of Cleaner Production. 2017;161:840-849.

[14] Boznar M, Lesjak M, Mlakar P. A neural network-based method for short-term predictions of ambient SO2 concentrations in highly polluted industrial areas of complex terrain. Atmospheric Environment. Part B. Urban Atmosphere. 1993;27(2):221-230.

[15] Gardner MW, Dorling SR. Artificial neural networks (the multilayer perceptron)—a review of applications in the atmospheric sciences. Atmospheric Environment. 1998;32(14-15):2627-2636.

[16] Qaderi F, Ayati B, Ganjidoust H. Role of moving bed biofilm reactor and sequencing batch reactor in biological degradation of formaldehyde wastewater. Journal of Environmental Health Science \& Engineering. 2011;8(4):295-306.

[17] Babanezhad E, Amini Rad H, Hosseini Karimi SS, Qaderi F. Investigating nitrogen removal using simultaneous nitrification-denitrification in transferring wastewater through collection networks with smalldiameter pipes. Water Practice and Technology. 2017;12(2):396-405.

[18] Qaderi F, Sayahzadeh AH, Azizi M. Efficiency optimization of petroleum wastewater treatment by using of serial moving bed biofilm reactors. Journal of Cleaner Production. 2018;192:665-677.

[19] Yavari SM, Qaderi F. Determination of thermal pollution of water resources caused by Neka power plant through processing satellite imagery. Environment, Development and Sustainability. 2018:1-23.

[20] Moghadam MT, Qaderi F. Modeling of petroleum wastewater treatment by Fe/Zn nanoparticles using the response surface methodology and enhancing the efficiency by scavenger. Results in Physics. 2019;15:102566-102576.

[21] Azizpour F, Qaderi F. Optimization, modeling and uncertainty investigation of phenolic wastewater treatment by photocatalytic process in cascade reactor. Environment, Development and Sustainability. (in press).

[22] Banimahd M, Yasrobi SS, Woodward PK. Artificial neural network for stress-strain behavior of sandy soils: Knowledge based verification. Computers and Geotechnics. 2005;32(5):377-386.

[23] Yeh IC. Modeling slump flow of concrete using second-order regressions and artificial neural networks. Cement and Concrete Composites. 2007;29(6):474-480.

[24] Babanezhad E, Qaderi F, Ziri MS. Spatial modeling of groundwater quality based on using Schoeller diagram in GIS base: a case study of Khorramabad, Iran. Environmental Earth Sciences. 2018;77(9):339.

[25] Sheikholeslami Z, Kebria DY, Qaderi F. Investigation of photocatalytic degradation of BTEX in produced water using $\gamma-\mathrm{Fe}_{2} \mathrm{O}_{3}$ nanoparticle. Journal of Thermal Analysis and Calorimetry. 2019;135(3):1617-1627.

[26] Nasiri EF, Kebria DY, Qaderi F. An experimental study on the simultaneous phenol and chromium removal from water using titanium dioxide photocatalyst. Civil Engineering Journal. 2018;4(3):585-593.

[27] Sheikholeslami Z, Kebria DY, Qaderi F. Nanoparticle for degradation of BTEX in produced water; an experimental procedure. Journal of Molecular Liquids. 2018;264:476-482.

[28] Asadi P, Rad HA, Qaderi F. Comparison of Chlorella vulgaris and Chlorella sorokiniana pa.91 in post treatment of dairy wastewater treatment plant effluents. Environmental Science and Pollution Research. 2019;26(28):29473-29489.

[29] Qaderi F, Sayahzadeh AH, Azizpour F, Vosughi P. Efficiency modeling of serial stabilization ponds in treatment of phenolic wastewater by response surface methodology. International Journal of Environmental Science and Technology. 2019;16(8):4193-4202.

[30] Tamadoni A, Qaderi F. Optimization of soil remediation by ozonation for PAHs contaminated soils. Ozone: Science \& Engineering. 2019;41(5): 454-472.

[31] Khalegh R, Qaderi F. Optimization of the effect of nanoparticle morphologies on the cost of dye wastewater treatment via ultrasonic/photocatalytic hybrid process. Applied Nanoscience. 2019:1-21

(C) 2019 by the author(s). This work is licensed under a Creative Commons Attribution 4.0 International License (http://creativecommons.org/licenses/by/4.0/). Authors retain copyright of their work, with first publication rights granted to Tech Reviews Ltd. 